\title{
Influencia del Y Plus en el Valor del Esfuerzo Cortante de Pared a través Simulaciones empleando Dinámica Computacional de Fluidos
}

\author{
Eddie F. Barrera*, Fredy A. Aguirre, Salvador Vargas y Edwin D. Martínez \\ Universidad Libre, Facultad. de Ingeniería, Dpto. Ingenieria Mecánica, Bogota D.C, Colombia \\ * Autor a quien debe ser enviada la correspondencia
}

Recibido Nov. 20, 2017; Aceptado Ene. 22, 2018; Versión final Mar. 28, 2018, Publicado Ago. 2018

\begin{abstract}
Resumen
En este trabajo se estudió la influencia del $Y$ plus $\left(\mathrm{y}^{+}\right)$sobre las fuerzas de fricción, mediante análisis computacional de fluidos, para evaluar el valor del esfuerzo cortante de pared $(\tau)$ y el coeficiente total de arrastre $\left(\mathrm{C}_{\mathrm{d}}\right)$ empleando un software comercial de dinámica de fluidos computacional, Ansys-Fluent v16.1. El análisis se realizó sobre la pared del casco de un vehículo autónomo submarino, con número de Reynolds de $1.36 \times 10^{6}$, evaluado con el diámetro del casco del submarino. Los resultados mostraron que aun cuando la variación del $\mathrm{Y}$ plus se encuentra dentro de la subcapa viscosa $\left(\mathrm{y}^{+}<2\right)$ existe una dependencia de los resultados de acuerdo con este parámetro. Para el mallado grueso $(\mathrm{y}+=1.55)$ y el mallado extra-fino $\left(\mathrm{y}^{+}=\right.$ 0.00375 ), el porcentaje de variación del esfuerzo cortante de pared, fue del $74 \%$, para el coeficiente de arrastre total fue 44,3\%; mientras que la variación del tiempo de simulación fue del $71 \%$.
\end{abstract}

Palabras clave: dinámica de fluidos computacional; esfuerzo cortante de pared; densidad de malla; Y plus; tiempo de simulación

\section{Influence of Y Plus on the value of the Wall Shear Stress and the Total Drag Coefficient through Computational Fluid Dynamics Simulations}

\begin{abstract}
In this work we studied the influence of $\mathrm{Y}$ plus $\left(\mathrm{y}^{+}\right)$on friction forces by computational fluid analysis to evaluate wall shear stress $(\tau)$ and the total drag coefficient $\left(\mathrm{C}_{\mathrm{d}}\right)$ using a commercial Computational Fluid Dynamics (CFD) software, ANSYS-FLUENT V16.1. The analysis was made on the hull wall of a submarine autonomous vehicle; the Reynolds number used was $1.36 \times 10^{6}$, based on the diameter of the submarine hull. The results showed that even though the $y^{+}$variation is within the viscous sublayer $\left(\mathrm{y}^{+}<2\right)$ there is a dependence of the shear wall stress and drag coefficient results according to this parameter. For coarse meshing $\left(y^{+}=1.55\right)$ and extra-fine meshing $\left(\mathrm{y}^{+}=0.00375\right)$; the percentage of variation of the wall shear stress was $74 \%$ and for the total drag coefficient was $44.3 \%$; while the variation of the simulation time was $71 \%$.
\end{abstract}

Keywords: computational fluid dynamics; wall shear stress; meshed density; $y+$; simulation time. 


\section{INTRODUCCIÓN}

La dinámica de fluidos computacional, en inglés Computacional Fluid Dynamics (CFD), inicia a principios de los años 1960s, pero sólo a principios de los años 1970s cuando un grupo de investigación dirigido por el Profesor Spalding el Imperial College en el Reino Unido inicia un proyecto ambicioso para simular flujos cortantes simples, Lauder y Spalding desarrollaron uno de los modelos RANS más usados en la industrial actualmente (k- $\varepsilon$ ); mientras que a finales de 1970 y principios de 1980 aparecen los primeros códigos de CFD (Abbes, 2017), (Symscape, 2017), desde entonces CFD ha tenido diferentes aplicaciones, por ejemplo, aerodinámica y aeronáutica (Johnson et al. 2005; Urkiolaa et al, 2017), vehículos submarinos autónomos hidrodinámicos (A.U.V por sus siglas en inglés) (Allstor et al, 2014; Xu y Ju, 2010); diseño aerodinámico de automóviles (Katz, 2006; Zannet et al, 2010) generación de energía (Chung, 2002; Akwa et al, 2002), en todas estas aplicaciones se han obtenido resultados exitosos, contribuyendo a la reducción de costos en los procesos de fabricación, mejora en el diseño y rediseño, y la optimización en el funcionamiento de los objetos de estudio. El análisis CFD se divide en tres fases principales, la primera es el preprocesamiento, la cual incluye la elección, construcción y tamaño del dominio computacional, condiciones de mallado y condiciones iniciales y de frontera la simulación, la segunda fase es el procesamiento y la tercera es el reporte y análisis de datos (Elhadi et al, 2012).

Es importante al momento de ejecutar el modelo de turbulencia, determinar el tamaño apropiado de las celdas cerca de las paredes del objeto de estudio, puesto que los resultados son sensibles al modelo seleccionado (Pope, 2016), durante la fase de pre procesamiento se debe garantizar que la calidad y la densidad de la malla sean adecuados, puesto que de esto dependerá la fiabilidad de los resultados, cabe resaltar la densidad de malla se define como el Número de elementos por unidad de área del mallado, y aunque Las mallas de mayor densidad suelen producir resultados de análisis más precisos requieren un mayor tiempo de análisis, es allí cuando surge la importancia del Y plus, el cual se define como una distancia adimensional, desde el punto donde se evalúa los parámetros que rigen la dinámica del flujo hasta la superficie del objeto de estudio, este se usa para describir cuantitativamente la densidad de la malla cerca de las paredes del objeto; al reducir la magnitud de $\mathrm{y}^{+}$, la distancia perpendicular de las celdas serán de menor tamaño, y por lo tanto si $y+\leq 5$, este se encuentra dentro la subcapa viscosa, donde los efectos de las fuerzas de fricción son de gran importancia (Baker, 2008; AIAA, 2008).

En esta investigación el objetivo principal, es analizar como el esfuerzo cortante sobre la pared $(\tau)$ y el coeficiente total de arrastre $\left(C_{d}\right)$ se ven afectados con la variación de $y^{+}$, la cual determina directamente la interacción entre el flujo y las paredes sólidas del objeto de estudio (González et al, 2011; Curtí, 2008; Mashud et al, 2008). Para verificar la precisión de los resultados utilizaron diferentes valores de $\mathrm{y}^{+}$: extra gruesa, gruesa, media, fina y extra-fina (NASA, 2015); debido a que en esta investigación se contemplan varios parámetros como el tipo de geometría, la clasificación del mallado, y los parámetros de estudio, se realizó la comparación del $\mathrm{C}_{d}$ con el valor determinado por la NASA experimentalmente en un túnel de viento, para un objeto de estudio con características geométricas y número de Reynolds similares (NASA, 2015).

\section{METODOLOGÍA}

Para estudiar la influencia del $\mathrm{y}^{+}$en el valor del esfuerzo cortante sobre pared y el coeficiente de arrastre total, es importante determinar la distancia perpendicular a la pared apropiada. Por otra parte, las simulaciones con mallas estructuradas son más eficientes en tiempo y resultados, sin embargo, la generación de este tipo de mallas requiere de gran inversión de tiempo; por lo cual, en este trabajo, únicamente se empleó una malla estructurada cerca de las paredes sólidas del objeto en estudio. El modelo de turbulencia empleado fue k-épsilon estándar.

\section{Análisis de Geometría}

La geometría utilizada en este estudio fue inicialmente planteada por Jourbert (2004), quien propuso tres tipos de formas geométricas diferentes, para los cascos de AUV, adecuadas para realizar un proceso de estudio; las formas se definen como, torpedo con punta radial, torpedo con punta elipsoidal y forma de gota. Para este trabajo se seleccionó el tipo de forma de gota, debido a que, de los tres tipos propuestos, esta forma presenta teóricamente el $\mathrm{C}_{d}$ más bajo, lo cual indica, una menor magnitud en la fuerza de arrastre hidrodinámico (Orrego et al, 2006; Jourbert, 2004). En la Figura 1 se presenta la geometría en 3D seleccionada. 


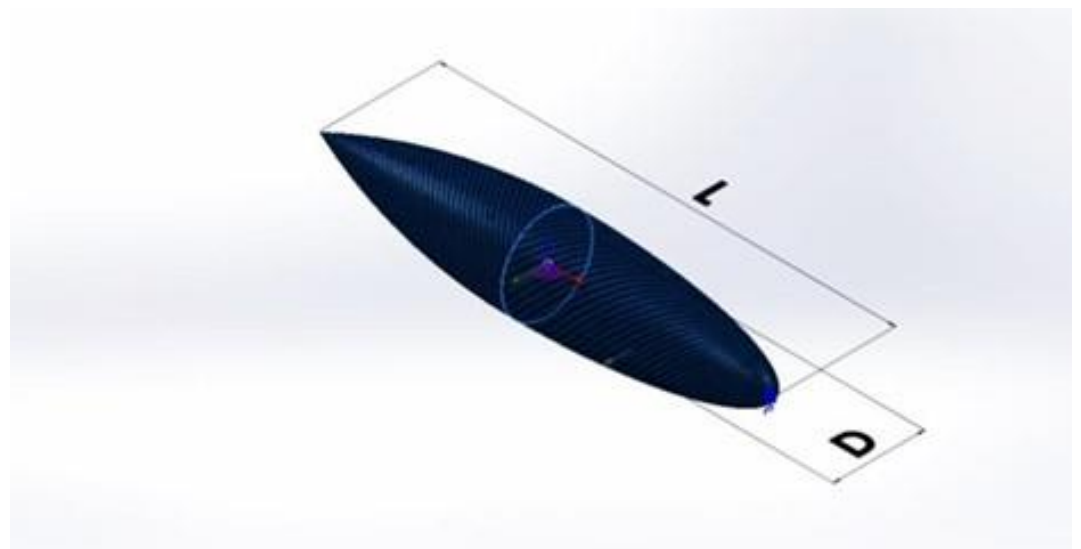

Fig.1: Geometría del casco en forma de gota

La fuerza total de arrastre posee dos componentes principales, la fuerza al arrastre superficial (fricción) y la fuerza por presión (forma), al incrementar la magnitud de la relación entre la longitud (L) y el diámetro del área de la sección transversal (D), la superficie aumenta, lo cual genera que la magnitud del arrastre superficial sea mayor, pero a su vez, la magnitud de la resistencia a la presión será menor; en general en los sólidos con forma revolucionada y simétricos, el efecto de reducir la relación (L/D), genera una disminución en la superficie, por lo tanto, un menor coeficiente de arrastre superficial. (Joubert, 2004; Aymed et al, 2017; Mashud, 2008).

Aguirre (2011) afirma que, para este tipo de sólido revolucionado y simétrico, como el empleado para este estudio, el punto de menor resistencia total al arrastre, se obtiene con $(L / D=5)$. Basándose en este criterio, y en el volumen útil dentro de este tipo de geometrías, el casco para este trabajo se diseñó con $L=1,8 \mathrm{~m}$ y $\mathrm{D}$ $=0,36 \mathrm{~m}$. (Acosta y Calvo, 2008)

\section{Modelamiento $3 D$}

El modelado de la geometría para este estudio se realizó en SolidWorks® 2015 , haciendo uso de las dimensiones reales para el diseño del casco hidrodinámico, las cuales fueron basadas en el criterio del volumen útil para el almacenamiento de componentes electrónicos dentro un AUV (Figura 1).

\section{Y plus y modelos matemáticos}

Varios factores determinan la calidad y la fiabilidad de los resultados de la simulación CFD, uno de ellos es $\mathrm{y}^{+}$, definido como una longitud adimensional; la cual es una distancia perpendicular desde el punto más cercano a la pared donde se evalúa la velocidad de fricción $\left(U_{*}\right)$, este parámetro se emplea para describir la resolución de la malla perpendicular a la pared solidad en estudio, matemáticamente se puede definir según la Ecuación 1.

$$
y^{+}=\frac{U_{*} \times d y}{v}
$$

En esta ecuación, $U_{*}$ es la velocidad de fricción en $(\mathrm{m} / \mathrm{s})$, dy es la distancia de la pared al punto donde se calculan los datos en este caso en $(\mathrm{m})$ y $\vee$ es la viscosidad cinemática del fluido en $\left(\mathrm{m}^{2} / \mathrm{s}\right)$.

La velocidad de fricción puede definirse, según la Ecuación 2.

$$
U_{*}=\sqrt{\frac{\tau}{\rho}}
$$

En esta ecuación, $\tau$ es el esfuerzo de corte en la pared en (Pa) y $\rho$ es la densidad del fluido en $\left(\mathrm{kg} / \mathrm{m}^{3}\right)$.

En 2008, la NASA convocó al $4^{\circ}$ taller de predicción de coeficiente de arrastre $\left(\mathrm{C}_{d}\right)$ de la Institución Americana de Aeronáutica y Astronáutica (AIAA) patrocinado por el comité técnico de aerodinámica aplicada (APA-TC) (AIAA, 2008) en donde se sugirió una clasificación del espaciamiento normal de malla dentro de la subcapa viscosa en función de $\mathrm{y}^{+}$como se muestra en la Tabla 1. 
Tabla 1: Clasificación de y+ según la NASA (datos tomados de AIAA, 2008)

\begin{tabular}{|c|c|}
\hline Rango de $y^{+}$ & Clasificación \\
\hline$y^{+}>2$ & Extra-gruesa \\
\hline $1>y^{+}>2 / 3$ & Gruesa \\
\hline $2 / 3>y^{+}>4 / 9$ & Media \\
\hline $4 / 9>y^{+}>8 / 27$ & Fina \\
\hline $8 / 27>y^{+}$ & Extra-fina \\
\hline
\end{tabular}

\section{Modelo de Turbulencia}

Hoy en día existen diferentes modelos de turbulencia, que se utilizan para el análisis hidrodinámico, entre los comunes y contemplados para realizar las simulaciones descritas en este trabajo, se encuentran el SpalartAllmaras (SA) y k-épsilon (k-e), sin embargo el SA al ser una modelo de una sola ecuación, en la que no se tiene en cuenta la energía cinética que produce la turbulencia, puede llegar a presentar en las zonas turbulentas un mayor margen de error, en aplicaciones aerodinámicos es lo suficientemente preciso sin embargo sus limitaciones con las zonas turbulentas lo aleja de ser considerado como un modelo general, por otro lado, k-épsilon tiene entre sus características, el ser un modelo de turbulencia de dos ecuaciones, donde k y e involucran la energía cinética debido a la turbulencia, lo cual permite mostrar efectos generados por esta, adicional, los cálculos convergen rápidamente y con precisión para diferentes regímenes de fluidos, útil cuando hay pequeños gradientes de presión y requiere de bajos recursos computacionales (Pope, 2016).

El modelo de turbulencia k-e debido a que aplica sus dos ecuaciones dentro de un régimen superior a la capa limite en el cual el fluido se torna turbulento, y puesto que requieren de bajos recursos computacionales en comparación a los modelos más avanzados, se analizó como la mejor opción de modelo de turbulencia para esta investigación (Bayona et al, 2015)

\section{Mallado}

El mallado, se clasifica en dos grandes grupos: malla estructurada (hexaedros) y mallas no estructurados (tetraedros, pirámides, prismas triangulares y poliedros) para geometrías tridimensionales; un tercer tipo de mallado aparece con la combinación de mallas estructuradas y no estructuradas, denominado mallado mixto o híbrido (Elhadi et al, 2012). En la figura 2, se observa el mallado predeterminado por el software, malla no estructurada en todo el dominio; por otro lado, en la figura 3 se presenta una malla híbrida; la cual cerca de la pared del objeto de estudio es estructurada y no estructurada en el resto del dominio; esto con el fin de tener control de la distancia perpendicular en esta zona.

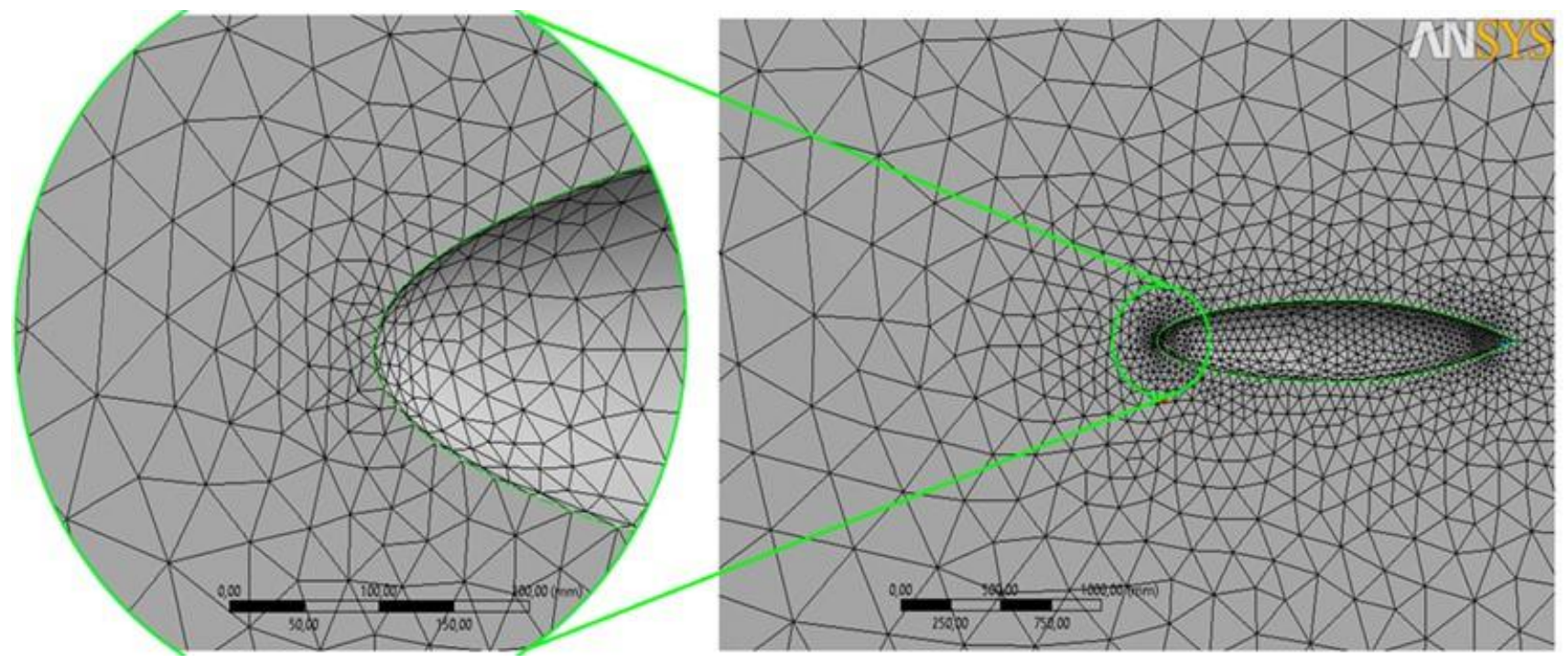

Fig. 2: Malla predeterminada por el software. 

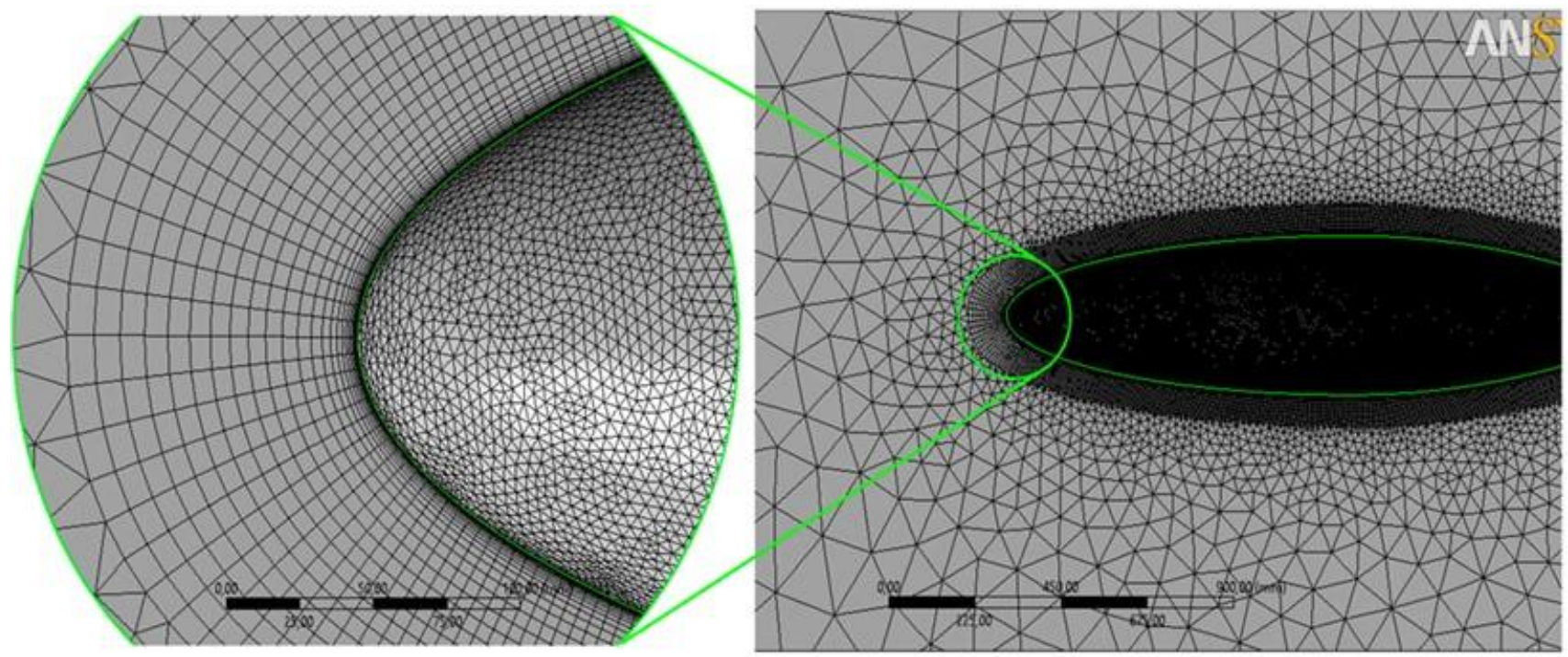

Fig. 3: Malla estructurada extra fina, $y^{+}<8 / 27$.

En este trabajo, a partir de la geometría tridimensional, Figura 1, se realizaron seis mallados diferentes incluyendo el mallado predeterminado por el software (Figure 2). La Tabla 2 muestra las características de cada uno de los las seis mallas.

Tabla 2: Características de cada mallado.

\begin{tabular}{|c|c|c|c|c|}
\hline \multirow[b]{2}{*}{ Mallado } & \multicolumn{2}{|c|}{ Capa límite } & \multirow{2}{*}{$\begin{array}{l}\text { Número de } \\
\text { Nodos en todo } \\
\text { el dominio }\end{array}$} & \multirow{2}{*}{$\begin{array}{l}\text { Número de } \\
\text { Elementos en } \\
\text { todo el dominio }\end{array}$} \\
\hline & $\begin{array}{c}\text { Número de capas } \\
\text { (malla } \\
\text { estructurada) }\end{array}$ & $\begin{array}{c}\text { Espesor de la capa } \\
\text { limite }(d y)(\mathrm{mm})\end{array}$ & & \\
\hline Predeterminada & - & - & 12646 & 64236 \\
\hline Extra gruesa & 10 & 0,15 & 487523 & 1964523 \\
\hline Gruesa & 30 & 0,015 & 695988 & 2366328 \\
\hline Media & 30 & 0,0064 & 839264 & 3216088 \\
\hline Fina & 40 & 0,0042 & 1438347 & 3539987 \\
\hline Extra fina & 40 & 0,0032 & 1836669 & 4310524 \\
\hline
\end{tabular}

\section{Calidad del mallado}

Existen varios parámetros que determinan la calidad de la malla, entre los más destacados y comúnmente analizados, se encuentran tres: Aspect ratio, es la proporción entre las dimensiones que poseen las celdas, Skewness el cual es la oblicuidad, medido en una escala de 0 a 1, siendo la tendencia a 0 para un mallado excelente, y por último, Orthogonal quality, analizado en una escala de 0 a 1 la tendencia a 1 es para un mallado perfecto (ANSYS, 2013). En la Tabla 3 se presentan los valores de los parámetros de calidad para cada uno de los mallados elaborados en este trabajo.

Tabla 3: Parámetros de análisis de calidad del mallado.

\begin{tabular}{|l|c|c|c|c|}
\hline \multicolumn{1}{|c|}{ Mallado } & $\begin{array}{c}\text { Minimum aspect } \\
\text { ratio }\end{array}$ & $\begin{array}{c}\text { Maximum aspect } \\
\text { ratio }\end{array}$ & $\begin{array}{c}\text { Skewness } \\
\text { average }\end{array}$ & Orthogonal quality average \\
\hline Preterminada & 1,1841 & 6,651 & 0,2447 & 0,8508 \\
\hline Extra gruesa & 1,1594 & 80,854 & 0,2236 & 0,8694 \\
\hline Gruesa & 1,1592 & 94,775 & 0,2045 & 0,8791 \\
\hline Media & 1,1590 & 130,400 & 0,1875 & 0,8939 \\
\hline Fina & 1,1588 & 1944,300 & 0,1566 & 0,9092 \\
\hline Extra fina & 1,1587 & 2978,4000 & 0,0742 & 0,9237 \\
\hline
\end{tabular}




\section{Descripción del problema}

La aplicación CFD permite analizar el comportamiento de la geometría bajo un contexto operacional, antes de la fabricación y puesta en funcionamiento de los diseños planteados, así como los resultados obtenidos de estos análisis, respaldan el trabajo desarrollado en la fase de diseño; sin embargo, está sujeto a la influencia de dos condiciones principales en la obtención de resultados, el tiempo necesario para alcanzar los resultados finales y la capacidad de los recursos computacionales que se posean; el uso de las herramientas proporcionadas por CFD, hace posible aprovechar al máximo los recursos computacionales sin sacrificar la fiabilidad de los resultados, el trabajo clave de esta investigación, es evaluar la influencia de la densidad de malla aplicada al esfuerzo cortante sobre la pared, en una geometría y condiciones de trabajo específicos, lo cual puede dar una aproximación de la influencia de $\mathrm{y}^{+}$en la fiabilidad de los resultados en un análisis CFD.(Garrido, 2015).

\section{Condiciones iniciales y de contorno}

El volumen de control o dominio se define como el límite del fluido en el que se sumerge el cuerpo de estudio. De acuerdo con la geometría seleccionada, el centro geométrico del casco está ubicado en las coordenadas $(0,0,0)$, desde este punto, la entrada de flujo aguas arriba al dominio, se colocó a una distancia de cinco veces la longitud de la geometría $(5 \mathrm{~L})$, la salida del flujo aguas abajo del dominio está se situó a una distancia de diez veces la longitud de la geometría (10L). Los límites superior, inferior, laterales se colocaron a una distancia de cinco veces el diámetro de la geometría (5D) (González, 2006). La Figura. 4 muestra la disposición de la geometría dentro del volumen de control.

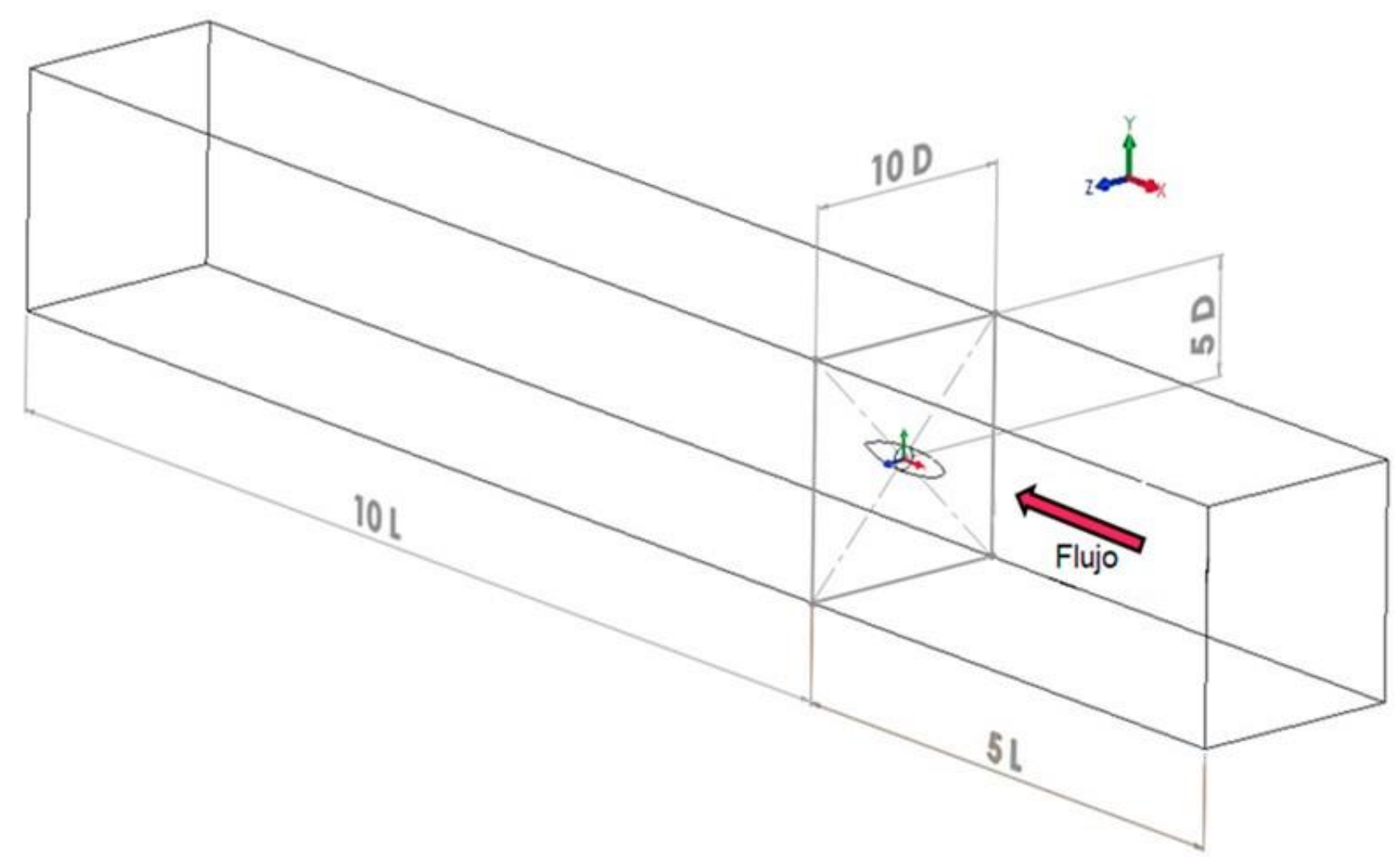

Fig. 4: Dimensiones del volumen de control

\section{Esquemas numéricos.}

Para este trabajo se empleó pressure velocity coupling con el esquema SIMPLE, los modelos de discretización empleados para la solución del problema fueron de segundo orden todos los parámetros. Las simulaciones numéricas se desarrollaron a una velocidad media formulada por Valdez (Valdez, 2010), quien realizó un estudio de los parámetros hidrodinámicos de la robótica submarina. El criterio de convergencia para todas las simulaciones numéricas de este estudio, fue del orden de $10^{-6}$, esta convergencia se obtuvo alrededor de 25000 iteraciones; cabe resaltar que las simulaciones se realizaron en estado permanente para las condiciones iniciales y despreciando los cambios generados a causa de la gravedad. 
Tabla 4: Parámetros de simulación.

\begin{tabular}{|l|c|}
\hline Velocidad media $(V)$ & $2 \mathrm{~m} / \mathrm{s}$ \\
\hline Número de Reynolds $(\mathrm{Re})$ & $1,63 \times 10^{6}$ \\
\hline Densidad $(\rho)$ & $998,2 \mathrm{~kg} / \mathrm{m}^{3}$ \\
\hline Presión $(\mathrm{P})$ & $2,06 \mathrm{MPa}$ \\
\hline Viscosidad dinámica $\mu$ & $0.00162 \mathrm{~kg} /(\mathrm{m} \cdot \mathrm{s})$ \\
\hline Temperatura $(\mathrm{T})$ & $3^{\circ} \mathrm{C}$ \\
\hline
\end{tabular}

\section{RESULTADOS}

En todas las gráficas, el eje de las abscisas los valores indican la posición geométrica a lo largo del objeto de estudio, el origen del sistema coordenado se encuentra ubicado el centro del objeto en estudio (Figura 4).. En la Figura 5, se presentan; los valores obtenidos para $\mathrm{y}^{+}$a lo largo de la longitud del objeto de estudio, la figura muestra un valor máximo de $\mathrm{y}^{+}=1000$, este valor es muy grande con respecto a la clasificación dada en la Tabla 1. En la Figura 6, se presentan los valores para los esfuerzos cortantes $(\tau)$ sobre la pared del casco hidrodinámico, la gráfica de la Figura 5 muestra valores alrededor de 0,1 $\mathrm{Pa}$.

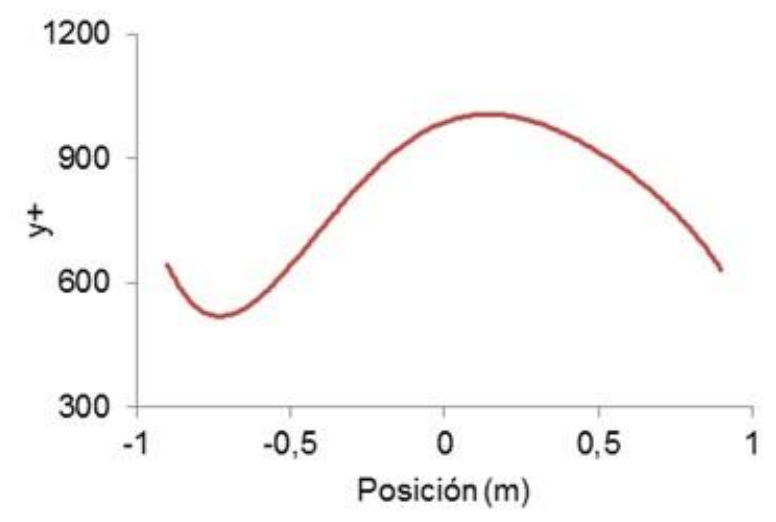

Fig. 5: Valores de $\mathrm{y}^{+}$para la malla predeterminada

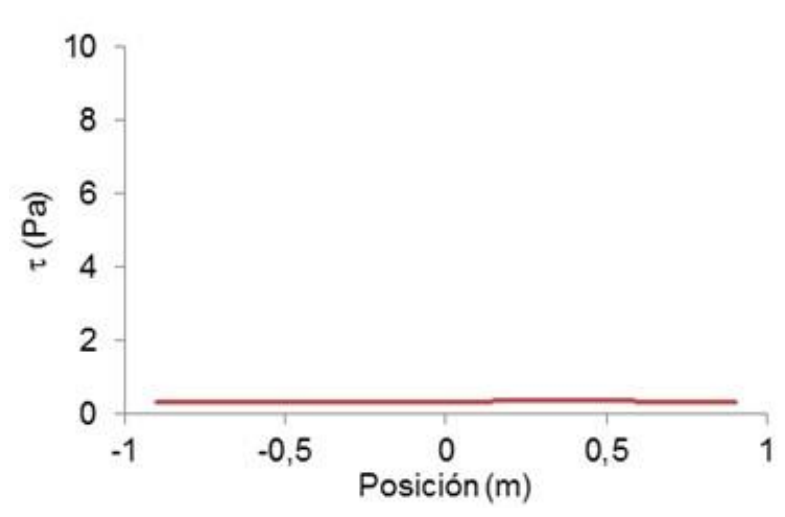

Fig. 6: Valores promedio de $\tau$ para la malla predeterminada

En los resultados obtenidos con mallas mixtas, se observó un patrón diferente de la curva de $\mathrm{y}^{+}$comparado el patrón obtenido con la malla predeterminado. Los patrones observados para todas las mallas mixtas son similares, valores mínimos en la parte posterior y valores máximos en la parte frontal del casco; La Figura 7 presenta los resultados obtenidos para y $^{+}$para una malla extra gruesa, el valor máximo obtenido fue de 2,25. En la Figura 8, se presentan los valores para los esfuerzos cortantes $(\tau)$ sobre la pared del casco hidrodinámico para una malla extra gruesa, el valor máximo presentado en la gráfica se encuentra alrededor de los 43,5 $\mathrm{Pa}$.

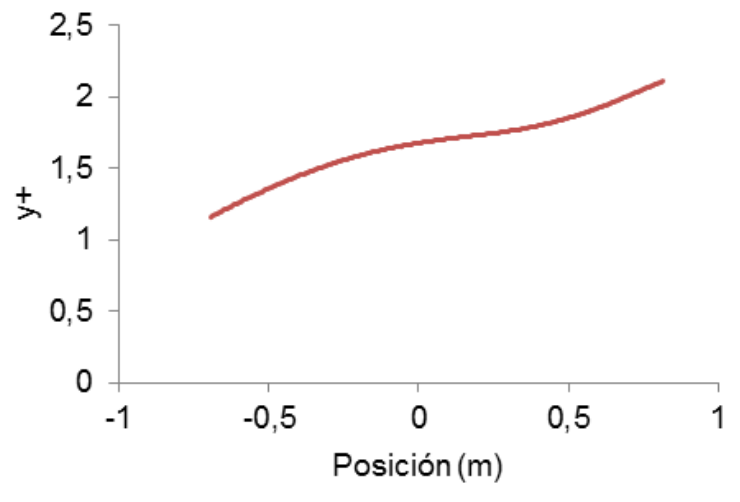

Fig. 7: Valores promedios de $\mathrm{y}^{+}$para malla extra gruesa

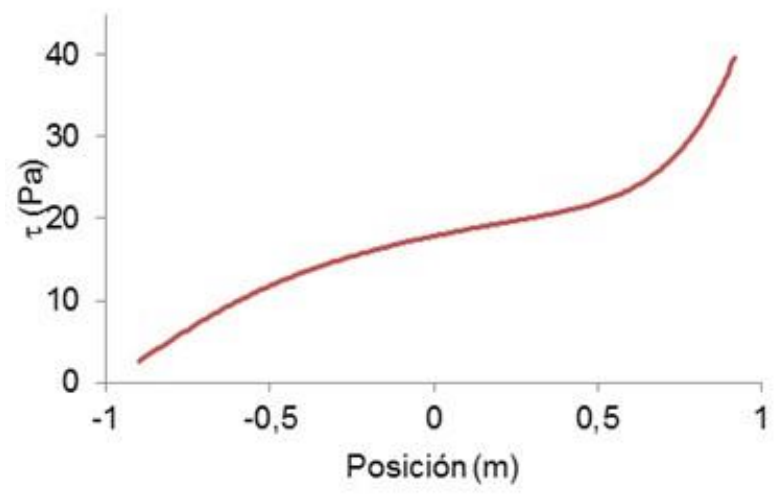

Fig. 8: Valores promedios de $\tau$ para una malla extra gruesa 
En la figura 9 se observan valores máximos de $\mathrm{y}^{+}$de aproximadamente 1.5, cerca de la parte frontal del casco; los esfuerzos cortantes sobre el casco se muestran en la Figura 10, el valor máximo está alrededor de $46 \mathrm{~Pa}$.

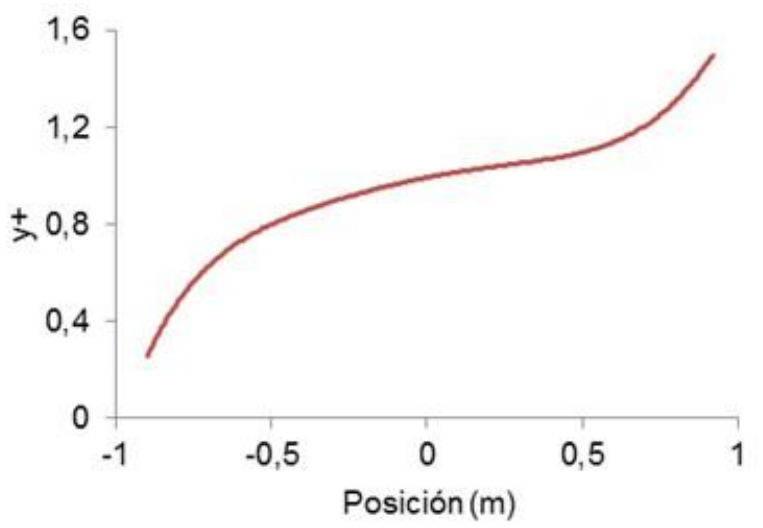

Fig. 9: Valores promedios del $\mathrm{y}^{+}$para una malla gruesa

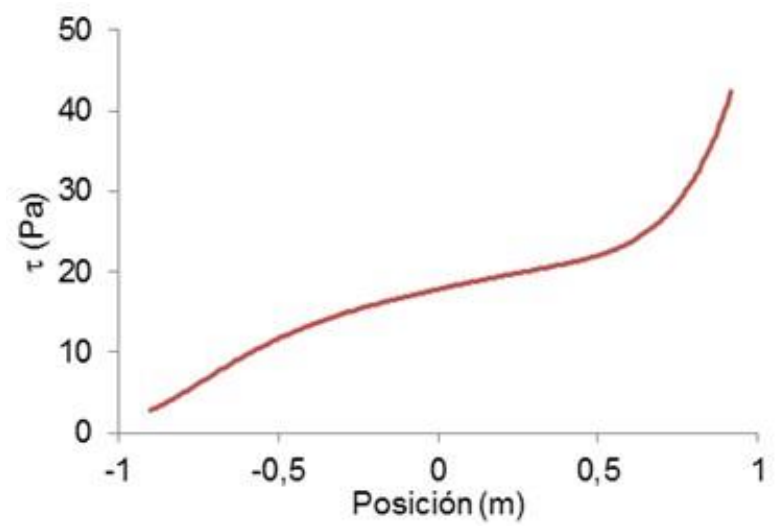

Fig.10: Valores promedios de $\tau$ en una malla media

La Figura 11, muestra un valor máximo de $\mathrm{y}^{+}=0.65$, este valor es clasificado en la Tabla 1 , como malla media. En la Figura 12 se observa el esfuerzo cortante sobre el casco, el valor máximo es de $45.9 \mathrm{~Pa}$, este valor es mayor en comparación con los valores anteriores.

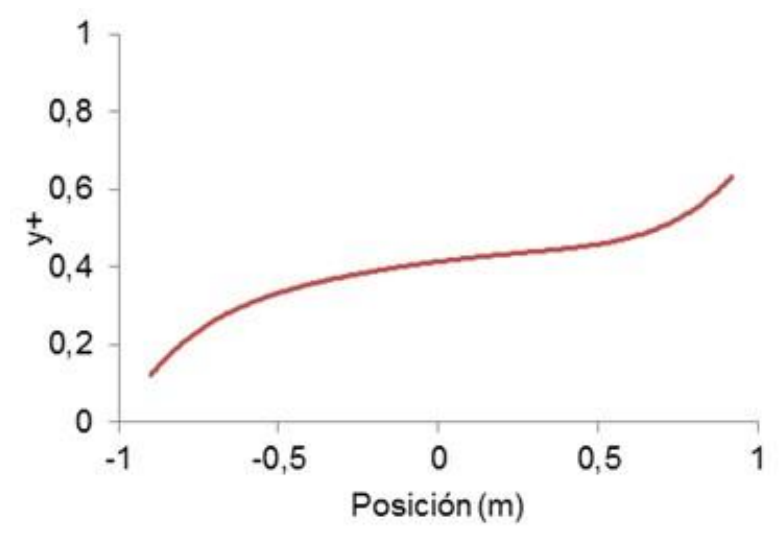

Fig. 11: Valores de $\mathrm{y}^{+}$en una malla media

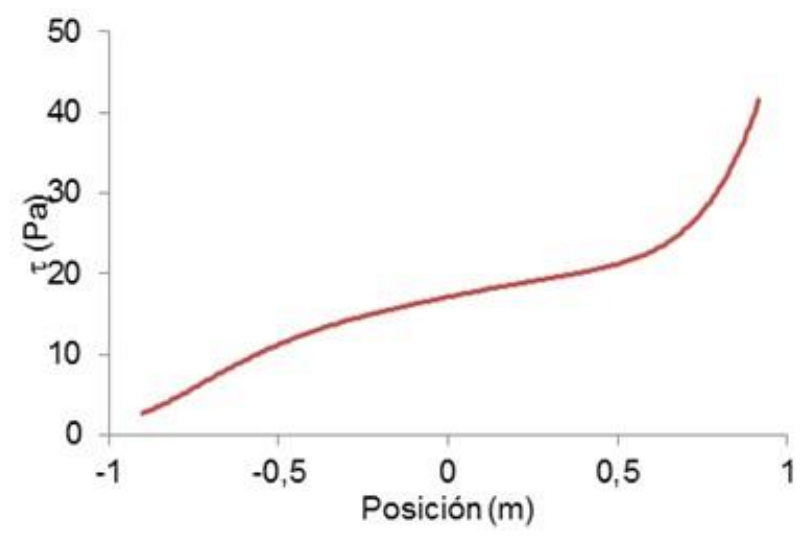

Fig. 12: Valores de $\tau$ en una malla media

La Figura 13 muestra que el valor máximo de $\mathrm{y}^{+}$disminuyeron hasta un valor de 0.425 , el cual se puede catalogar como malla fina según la Tabla 1. La Figura 14 muestra valores de esfuerzos cortantes sobre el objeto de estudio, el valor máximo de $\tau$ es $44.75 \mathrm{~Pa}$.

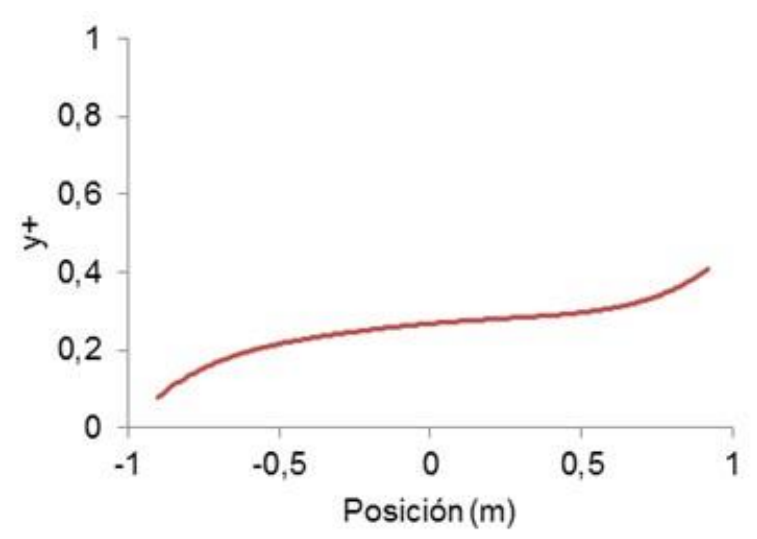

Fig. 13 Valores $\mathrm{y}^{+}$en una malla fina.

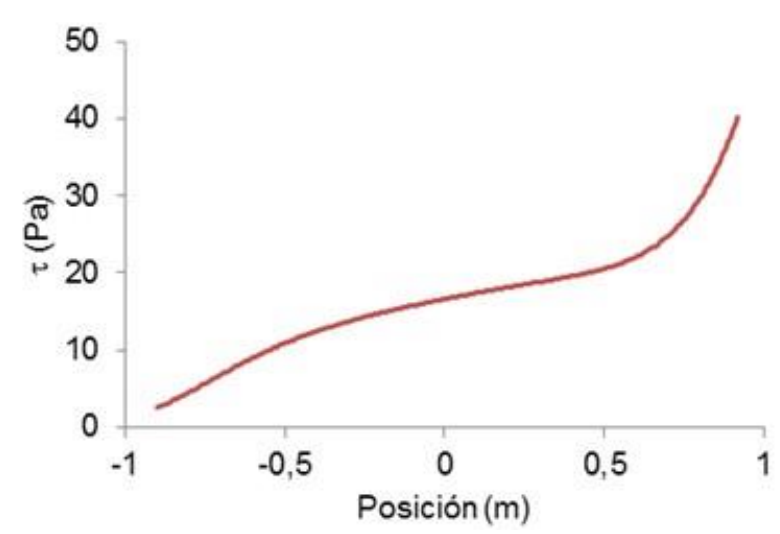

Fig. 14: Valores de $\tau$ en una malla fina.

Para esta simulación el valor máximo $\mathrm{y}^{+}$es del orden de 0.00375 , tal como se muestra en la Figura 15 , de acuerdo con este valor esta malla se puede clasificar como una malla extra fina según lo propuesto en la Tabla 1. La figura. 16 muestra que el valor máximo del esfuerzo cortante sobre el casco para una malla extrafina, el cual es de $25 \mathrm{~Pa}$, se observa un incremento con respecto a la malla fina. 


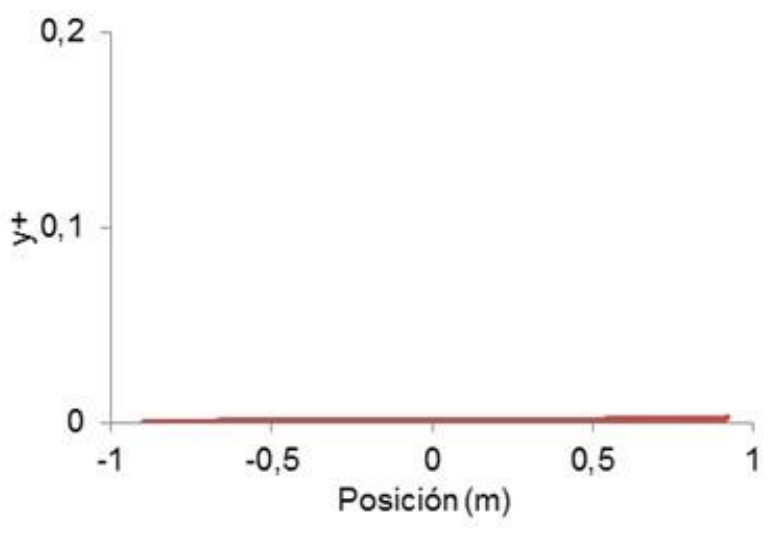

Fig. 15: Valores $\mathrm{y}^{+}$en una malla extrafina.

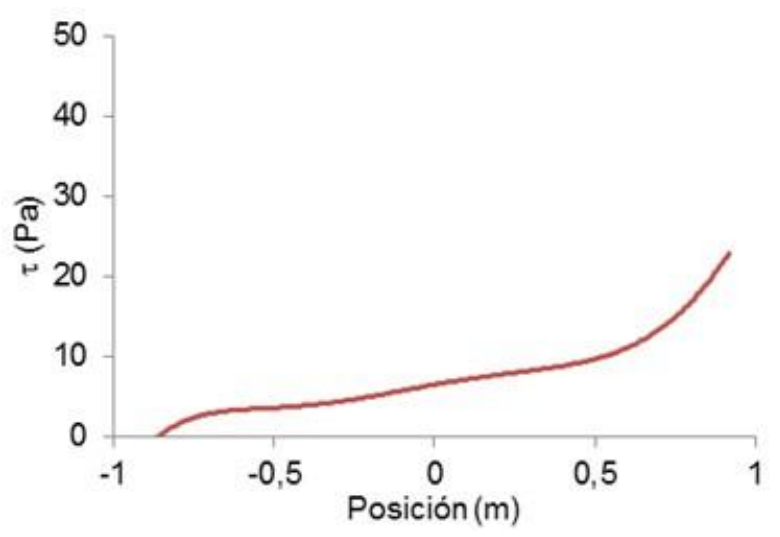

Fig. 16: Valores de $\tau$ en una malla extrafina

\section{ANÁLISIS DE RESULTADOS}

Los resultados obtenidos tanto de $\mathrm{y}^{+}$como de $\tau$ se graficaron con respecto a la longitud de la geometría de estudio para las seis diferentes valores de $\mathrm{y}^{+}$según la Tabla 1. Los resultados obtenidos para la malla por defecto mostraron valores de $\mathrm{y}^{+}$extremadamente grandes, alrededor de 500 veces lo establecido para una malla gruesa según la Tabla 1, y un esfuerzo cortante sobre la pared con un valor aproximado de $0,1 \mathrm{~Pa}$, es decir 250 veces más pequeño en comparación al valor generado para una malla extrafina. Estos resultados sugieren que los datos generados con valores de $\mathrm{y}^{+}$grandes, tienen una variación alta de esfuerzo cortante aún para una malla de clasificación gruesa, por esta razón estos valores no fueron tomados en cuenta para un comparativo entre las mallas.

En la Tabla 5 se presenta un resumen de los resultados para $\mathrm{y}^{+}, \square$ y $\mathrm{C}_{\mathrm{d}}$; para cada una de las mallas analizadas en este trabajo, adicional se evaluó el porcentaje de error del coeficiente de arrastre en relación al valor de referencia, tomado según un estudio realizado por la NASA en el año 2015 y que tenía el fin de determinar experimentalmente los coeficientes de arrastre de distintas geometrías, entre estas, para un cuerpo de geometría y número de Reynolds similares al objeto de estudio, éste valor fue determinado experimentalmente mediante la utilización de un modelo en un túnel de viento y tuvo un resultado de $C_{d}=$ 0,045 (NASA, 2015).

Tabla 5: Resumen de resultados

\begin{tabular}{|c|c|c|c|c|c|c|}
\hline Clasificación & $\begin{array}{c}\text { Espesor de la capa } \\
\text { limite (dy) }\end{array}$ & $y^{+}$ & $\tau$ & $C_{d}$ & $C_{d}-N A S A$ & \% Error \\
\hline Extra gruesa & 0,15 & 3,88 & 43,5 & 0,02186 & \multirow{5}{*}{0,045} & $51 \%$ \\
\hline Gruesa & 0,015 & 1,55 & 45,9 & 0,02197 & & $51 \%$ \\
\hline Media & 0,0064 & 0,65 & 44,75 & 0,02242 & & $50 \%$ \\
\hline Fina & 0,0042 & 0,425 & 42,5 & 0,02322 & & $48 \%$ \\
\hline Extra fina & 0,0032 & 0,00375 & 25 & 0,03948 & & $12 \%$ \\
\hline
\end{tabular}

En la Figura 17 se representan los datos de $\tau$ y $\mathrm{y}^{+}$obtenidos para las diferentes mallas: extra-gruesa, gruesa, medias, fina y extra-fina. La gráfica muestra que a medida que $\mathrm{y}^{+}$disminuye desde 1.55 hasta 0.00375 , el esfuerzo cortante de pared también disminuye desde un valor de 45,9 Pa hasta $25 \mathrm{~Pa}$; esta disminución en el esfuerzo cortante representa un $46.4 \%$, cuando se analiza un parámetro tan importante como lo es el esfuerzo cortante de pared, el cambio de $\mathrm{y}^{+}$juega un papel muy importante en los resultados finales; un claro ejemplo es el cálculo del coeficiente de arrastre total, lo cual podría suponer una selección errónea de la fuente de potencia para poder mover este objeto y consecuentemente un incremento en el consumo de combustible, ya que estos parámetros dependen directamente del esfuerzo cortante y la presión sobre el objeto en estudio.

Por otro lado, para poder validar los resultados de este trabajo, se evaluó la dependencia del coeficiente de arrastre total debido a $\mathrm{y}^{+}$, obteniéndose los siguientes porcentajes de diferencia entre mallas: $0.5 \%$ entre la malla extra gruesa y la malla gruesa; $2 \%$ entre la malla gruesa y la malla media; $3,4 \%$ entre la malla media y la malla fina y por último el $41,19 \%$ entre la malla fina y la malla extra fina. Por otro lado, la reducción porcentual para cada una de las transiciones de $\mathrm{y}^{+}$es la siguiente: extra gruesa y gruesa $60,1 \%$, gruesa y media $58,1 \%$, media y fina $34,6 \%$ y entre fina a y extrafina $99,1 \%$. El coeficiente de arrastre como una función del tipo de malla se muestra en la gráfica de a Figura 18. 


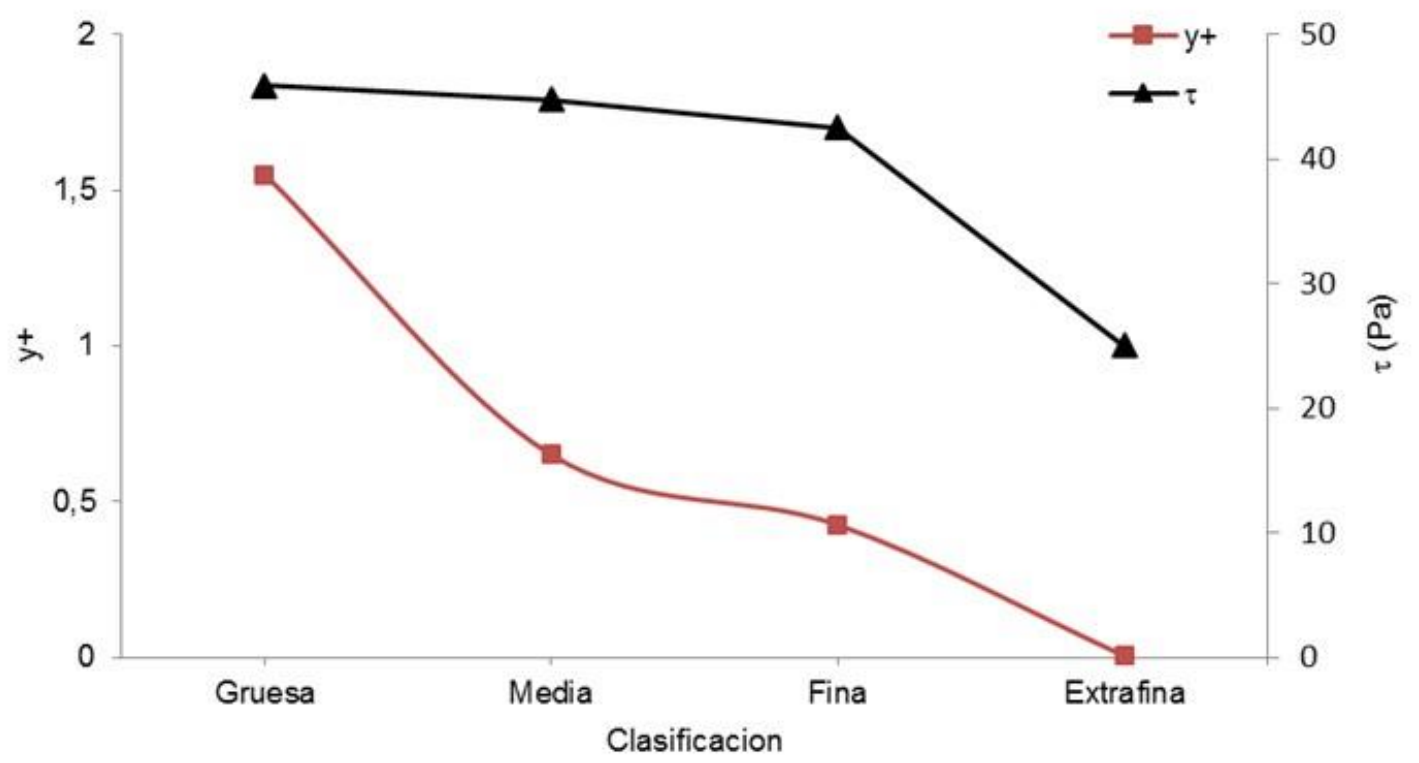

Fig. 17: Variación de $y+y \tau$ versus nivel de resolución de malla

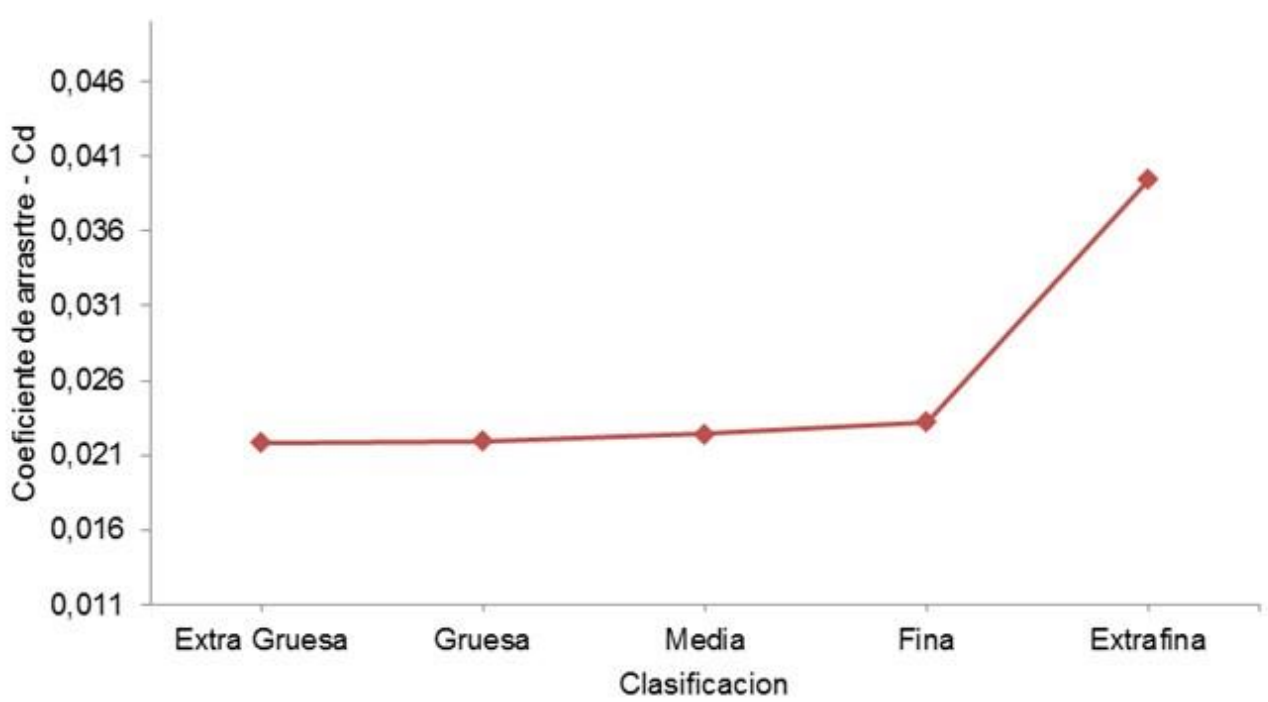

Fig. 18: Coeficiente de arrastre total versus resolución de malla

En la Tabla 6 se presentan los tiempos de simulación en horas de acuerdo con el nivel de resolución de la malla cerca de la pared basada en la distancia perpendicular adimensional, $\mathrm{y}^{+}$. Como se observa en la Tabla 6 , al disminuir el valor de $y^{+}$en la zona subviscosa, el tiempo de simulación se incrementa; en la Figura 19, se muestran gráficamente los datos de la Tabla 6 . En esta gráfica se observa el siguiente incremento porcentual en tiempo de simulación: entre la malla gruesa y la malla media fue del $42,5 \%$, entre la media y la malla fina el incremento fue de $25 \%$, para la malla fina y la malla extra fina el porcentaje de diferencia fue del $38,5 \%$. Las series de simulaciones se realizaron en un ordenador portátil con las siguientes características: Sistema operativo Windows de 10 a 32 bits, procesador AMD E-450 - $1.6 \mathrm{GHz}$, memoria RAM de $2048 \mathrm{MB}$, GPU gráficos AMD Radeon ${ }^{\mathrm{T} M}$ y HD 6320.

Tabla 6: Datos concluyentes de las simulaciones para cada tipo de malla

\begin{tabular}{|c|c|c|c|c|}
\hline Clasificación & Gruesa & Media & Fina & Extra fina \\
\hline$y_{+}$ & 1,55 & 0,65 & 0,425 & 0,00375 \\
\hline Tiempo $(h)$ & 3,45 & 6 & 8 & 13 \\
\hline
\end{tabular}




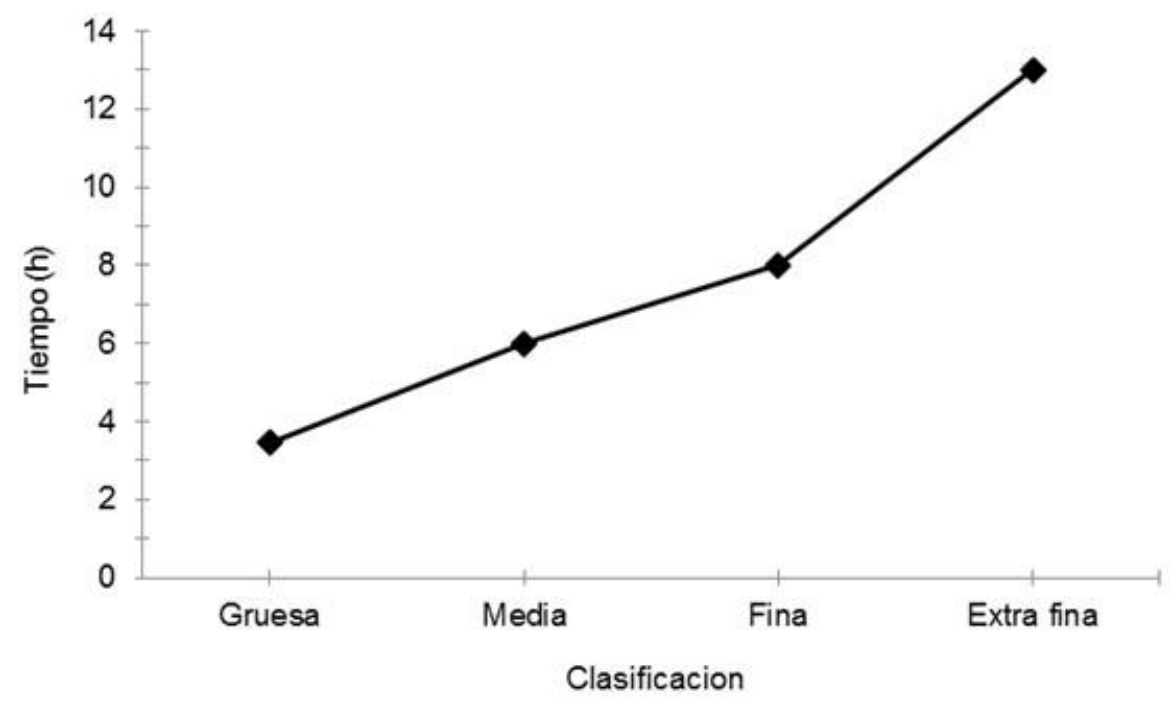

Fig. 19: Tiempo de simulación versus clasificación mallado

\section{CONCLUSIONES}

Este trabajo presentó un estudio de densidad de malla basado en el parámetro adimensional y+ sobre una geometría de casco submarino con forma de gota simétrica en cuanto al eje axial, y donde se buscó determinar los efectos del valor de y+ en el esfuerzo cortante de pared y el coeficiente total de arrastre. El estudio de densidad de malla se desarrolló aplicando un modelo de turbulencia k- $\varepsilon$ standard, y se evaluó el tiempo de simulación para estimar la mejor opción entre los parámetros analizados.

Los resultados mostraron que para un cambio entre una mallado grueso a extra fino, se aumentó en un $73,5 \%$ en el tiempo de simulación, el esfuerzo cortante sobre la pared vario en $46,4 \%$ y la variación del coeficiente total de arrastre entre un mallado grueso y un extra fino es de $44.36 \%$. Estos resultados muestran claramente que si estamos dentro de la subcapa viscosa, el esfuerzo cortante de la pared tiene un cambio menor, pero el cual podría ser muy importante en los resultados finales si los parámetros estudiados están directamente involucrados con el esfuerzo cortante de la pared; tales como fuerzas de fricción por arrastre.

Una conclusión final obtenida de este trabajo sugiere que si se necesita obtener datos confiables, cercanos a la pared del objeto de estudio, debe usar una malla de densidad extra fina, debido a que el cambio de un mallado fino a extrafino, genero un $41,19 \%$ de cambio para el coeficiente total de arrastre, mientras que se necesita un $38,5 \%$ más en el tiempo de simulación.

\section{AGRADECIMIENTOS}

Los autores agradecen el apoyo de la Universidad Libre y al grupo de investigación DETECAL del programa de Ingeniería Mecánica, para el desarrollo de este trabajo de investigación.

\section{REFERENCIAS}

Abbes A., A Brief History of Fluent (2017)

Acosta, G. y O. Calvo, Vehículo Autónomo Submarino para la Inspección de Tuberías y Cables, Universidad Nacional del Sur, Argentina (2008)

Aguirre, F.A., F. Grau y J. Tornero, Optimal Design Parameters of AUV Hull Based on CFD Simulation, Instrumentation viewpoint, 11,18-18 (2011)

Akwa, J.V., H.A. Vielmo y A.P. Petry, A review on the performance of Savonius wind turbines, Renewable and Sustainable Energy, 16(5), 3054-3064 (2002)

Allstor, T., J. Munroe y otros cuatro autores, Predicting the wake behind a large AUV hydrofoil, Methods in Oceanography, 10, 166-177 (2014)

AIAA, $4^{\text {th }}$ AIAA CFD Drag Prediction Workshop Gridding Guidelines, American Institute of Aeronautics and Astronautics (2008)

ANSYS, ANSYS HELP, Mesh Quality (2013)

Aymed, M., K. Hedi y otros dos autores, Impact of stern design on hydrodynamic drag of AUV's hull, Indian journal of geo marine sciences, 47(1), 89-95 (2017) 
Baker, C., Estimating Drag Forces on Submarine Hulls, University of New Brunswick Defence, Canada (2008)

Barbera, A., Diseño de formas y análisis de resistencia de un vehículo autónomo submarino, Escuela superior de ingeniería naval y oceánica, Universidad Politécnica de Cartagena, Colombia (2013)

Bayona, C., L. Londoño, E. Nieto, Identificación del modelo de turbulencia más adecuado, utilizando software de dinámica de fluidos computacional, para el diseño del vehículo urbano de la competencia Shell Eco Marathon, Tesis, Universidad de San Buenaventura, Colombia (2015)

Chung, T.J., Computational Fluid Dynamics, University Press, ${ }^{\text {da }}$ Ed., 13-23, Cambridge (2002)

Cooper, G. E., Resource Management on the Flight Deck, National Aeronautics and Space Administration, Ames Research Center, 2120 (1980)

Curtí, H., Estrategia de Búsqueda y Seguimiento de Objetos Sobre el Lecho Marino con un Vehículo Submarino Autónomo, Universidad Nacional del Centro de la Provincia, Argentina (2008)

Elhadi, G.R., J.B. Ali y K. Evangelos, Una Investigación de CutCell Mallado Estrategias para la Predicción Precisa el Rendimiento Aerodinámico, SAE International, Journal of Turismos-Mecánicos Sistemas, 5, 369-380 (2012)

Garrido, A., Estimación de los Coeficientes Hidrodinámicos de Vehículos Autónomos Submarinos Mediante CFD, Universidad Politécnica de Cartagena, Unidad de Tecnología Naval, Colombia (2015)

González, A., T. Maestre y otros cuatro autores, Vehículo Submarino Autónomo para Trabajos Oceanográficos en Aguas Costeras, NOAA, Noviembre (2011)

González, J., AUV Multipropósito de Bajo Consumo (II), Ingeniería Naval, La Coruña, España (2006)

Johnson, F.T., E.N. Tinoco y N. J. Yu, Thirty years of development and application of CFD at Boeing Commercial Airplanes, Computers and Fluids, 34(10), 1115-1151, Seattle (2005)

Joubert, P. N., Some Aspects of Submarine Design Part 1, Hydrodynamics, Public Release, Australia (2004)

Katz, J., Aerodynamics of race cars, Annual Review of fluid mechanics, 38, 27-63 (2006)

Mashud, M., R. Mahbudar y A. Alim, Numerical computation of viscous drag for axisymmetric underwater vehicles, Journal Mekanikal, 26, 9 - 21 (2008)

NASA, Shape effects on drag, National Aeronautics and Space Administration, Glenn research center (2015)

Orrego, S., A. Bernal y M. García, Diseño de un Ala para un Vehículo Aéreo no Tripulado Usando CFD, Univ. Eafit (2006)

Pope, S., Turbulent flows, Cambridge, Cambridge University Press (2016)

Symscape, Origins of the commercial of CFD industry (2017)

Urkiolaa, A., U. Fernandez-Gamiz, I. Erraste y E. Zulueta, Computational characterization of the vortex generated by vortex generator on a flat plate for different vane angles, Aerospace, Sci. Technol., 65,18-25 (2017)

Valdez, D., Estado del Arte en Robótica Submarina, Tesis de Maestría en Ciencias, Univ. de Valencia, España (2010)

Xu, Z.H. y C.H. Ju, Using CFD software to calculate hydrodynamic coefficients, Marine, Sci. Appl., 9(2), 149-155 (2010)

Zannet, J., D. Imbault y A.Tourabi, A design methodology for cross water turbines, Renewable ENERGY, 35(5), 9971009 (2010) 